\title{
Development and Characterization of Nb3Sn/Al203 Superconducting Multilayers for High-performance Radio-Frequency Applications
}

\section{Chris Sundahl}

University of Wisconsin-Madison

Junki Makita

Old Dominion University

Paul Welander

SLAC National Accelerator Laboratory

Yi-Feng Su

Florida State University

Fumitake Kametani

Florida State University

Lin Xie

Southern University of Science and Technology

Huimin Zhang

West Virginia University

Alex Gurevich

Old Dominion University

Chang-Beom Eom (D EOM@ENGR.WISC.EDU)

University of Wisconsin-Madison

Lian Li

West Virginia University

\section{Research Article}

Keywords: Superconducting radio-frequency (SRF), high-energy particles, magnetic fields, superconductors

Posted Date: January 22nd, 2021

DOI: https://doi.org/10.21203/rs.3.rs-151336/v1

License: (a) (i) This work is licensed under a Creative Commons Attribution 4.0 International License.

Read Full License 
Version of Record: A version of this preprint was published at Scientific Reports on April 8th, 2021. See the published version at https://doi.org/10.1038/s41598-021-87119-9. 
Development and characterization of $\mathrm{Nb}_{3} \mathrm{Sn} / \mathrm{Al}_{2} \mathrm{O}_{3}$ superconducting multilayers for highperformance radio-frequency applications

Chris Sundahl ${ }^{1}$, Junki Makita ${ }^{2}$, Paul B. Welander ${ }^{3}$, Yi-Feng Su ${ }^{4}$, Fumitake Kametani ${ }^{4,5}$, Lin Xie ${ }^{6}$, Huimin Zhang ${ }^{7}$, Lian $\mathrm{Li}^{7}$, Alex Gurevich ${ }^{2 *}$, Chang-Beom Eom ${ }^{1 *}$

Superconducting radio-frequency (SRF) resonator cavities provide extremely high quality factors $>10^{10}$ at $1-2 \mathrm{GHz}$ and $2 \mathrm{~K}$ in large linear accelerators of high-energy particles. The maximum accelerating field of SRF cavities is limited by penetration of vortices into the superconductor. Present state-of-the-art $\mathrm{Nb}$ cavities can withstand up to $50 \mathrm{MV} / \mathrm{m}$ accelerating gradients and magnetic fields of 200-240 $\mathrm{mT}$ which destroy the low-dissipative Meissner state. Achieving higher accelerating gradients requires superconductors with higher thermodynamic critical fields, of which $\mathrm{Nb}_{3} \mathrm{Sn}$ has emerged as a leading material for the next generation accelerators. To overcome the problem of low vortex penetration field in $\mathrm{Nb}_{3} \mathrm{Sn}$, it has been proposed to coat $\mathrm{Nb}$ cavities with thin film $\mathrm{Nb}_{3} \mathrm{Sn}$ multilayers with dielectric interlayers. Here, we report the growth and multi-technique characterization of stoichiometric $\mathrm{Nb}_{3} \mathrm{Sn}_{/} \mathrm{Al}_{2} \mathrm{O}_{3}$ multilayers with good superconducting and $\mathrm{RF}$ properties. We developed an adsorption-controlled growth process by co-sputtering $\mathrm{Nb}$ and $\mathrm{Sn}$ at high temperatures with a high overpressure of $\mathrm{Sn}$. The cross-sectional scanning electron transmission microscope images show no interdiffusion between $\mathrm{Al}_{2} \mathrm{O}_{3}$ and $\mathrm{Nb}_{3} \mathrm{Sn}$. Low-field RF measurements suggest that our multilayers have quality factor comparable with cavitygrade $\mathrm{Nb}$ at 4.2 $\mathrm{K}$. These results provide a materials platform for the development and optimization of high-performance SIS multilayers which could overcome the intrinsic limits of the $\mathrm{Nb}$ cavity technology.

${ }^{1}$ Department of Materials Science and Engineering, University of Wisconsin-Madison, Madison, WI 53706. ${ }^{2}$ Physics Department and Center for Accelerator Science, Old Dominion University, Norfolk, VA 23529. ${ }^{3}$ SLAC National Accelerator Laboratory, Menlo Park, CA 94025. ${ }^{4}$ Applied Superconductivity Center, National High Magnetic Field Laboratory, Florida State University. ${ }^{5}$ Department of Mechanical Engineering, FAMU-FSU College of Engineering, Tallahassee, FL 32310. ${ }^{6}$ Department of Physics, Southern University of Science and Technology, Shenzhen, 518055, China. ${ }^{7}$ Department of Physics and Astronomy, West Virginia University, Morgantown, WV 26506.

*eom@engr.wisc.edu, agurevic@odu.edu 


\section{Introduction}

For decades, $\mathrm{Nb}$ has been the material of choice for the radio-frequency superconducting (SRF) resonators for high-energy particle accelerators. Technological advances have resulted in the development of $\mathrm{Nb}$ cavities which can exhibit extremely high quality factors $\mathrm{Q}>10^{10} @ 1-2 \mathrm{GHz}$ and $2 \mathrm{~K}$ while sustaining accelerating gradients up to $50 \mathrm{MV} / \mathrm{m}^{1-3}$. Such exemplary performance and low RF losses can only be achieved if the cavities operate in a Meissner state which can persist up to the maximum magnetic field at the inner cavity surface reaches the superheating field $\mathrm{B}_{\mathrm{s}}=$ $240 \mathrm{mT}^{1-3}$. At $\mathrm{B}=\mathrm{B}_{\mathrm{s}}$ the low-dissipative Meissner state becomes absolutely unstable with respect to dissipative penetration of vortices, causing an explosive increase of RF power and thermal quench of the cavity. The state-of-the-art $\mathrm{Nb}$ cavities can already operate at the peak magnetic field close to $\mathrm{B}_{\mathrm{s}}$, thus, increasing accelerating gradients beyond the intrinsic limits of $\mathrm{Nb}$ requires materials with higher $B_{s}$. There are many such materials but all of them are type-II superconductors with lower critical field $\mathrm{B}_{c 1}$ smaller than $B_{c 1} \approx 170-180 \mathrm{mT}$ of $\mathrm{Nb}$ which makes high-B $\mathrm{B}_{\mathrm{s}}$ superconductors prone to detrimental penetration of vortices at low fields ${ }^{4,5}$. To overcome this problem, it was proposed to nanostructure the inner surface of $\mathrm{Nb}$ cavities by coating it with multilayers of thin superconductors $(\mathrm{S})$ separated by dielectric insulating (I) layers (

Figure 1$)^{6}$. Here the $\mathrm{S}$-layer material has a superheating field $\mathrm{B}_{\mathrm{s}}$ higher than $\mathrm{B}_{\mathrm{s} 0}$ of $\mathrm{Nb}$, whereas the thickness $d$ of S layers is smaller than the London penetration depth $\lambda$, and the thickness of I layers can be a few nm to suppress the interlayer Josephson coupling. Such SIS structures greatly increase barriers for penetration of vortices in the bulk of the cavity which could potentially withstand the RF fields limited by the superheating field of S-layer. For instance, using $\mathrm{Nb}_{3} \mathrm{Sn}$ with $B_{S}=480 \mathrm{mT}$ could nearly double the maximum accelerating gradient as compared to the best $\mathrm{Nb}$ cavities. The multilayer approach is based on the lack of thermodynamically stable parallel vortices in thin decoupled S screens at $B<B_{c 1}$ where $B_{c 1}$ is strongly enhanced in films with $d<$ $\lambda^{6-10}$. Because the inner surface of the $\mathrm{Nb}$ cavity is partially screened by multilayers, both $\mathrm{Q}(\mathrm{H})$ and the breakdown field can be increased due to lower surface resistance $\mathrm{R}_{\mathrm{s}}$ and higher $\mathrm{H}_{\mathrm{c}}$ of the layer material ${ }^{6}$.

The multilayer coating, which opens up a principal opportunity to break the Nb monopoly in SRF cavities, has been tested by several groups using $\mathrm{MgB}_{2}, \mathrm{Nb}_{3} \mathrm{Sn}, \mathrm{NbN}, \mathrm{NbTiN}$, and dirty $\mathrm{Nb}$ as coating materials. These experiments have shown an increase of the dc field onset of penetration of vortices on $\mathrm{Nb}$ surfaces coated with different SIS structures ${ }^{4,11-19}$, although such key SRF characteristics as the surface resistance and quality factors of SIS multilayers under high-amplitude $\mathrm{RF}$ fields have been investigated to a much lesser extent. The first results on low-field $\mathrm{Q}$ measurements on $\mathrm{NbN} / \mathrm{MgO}$ multilayers ${ }^{13,19}$ have shown that SIS multilayers can have lower $\mathrm{R}_{\mathrm{s}}$ than bulk $\mathrm{Nb}$. However, the $\mathrm{SRF}$ performance of $\mathrm{Nb}_{3} \mathrm{Sn}$, the current material of choice for the next generation coating material ${ }^{20}$, has not yet been investigated in SIS structures. The development of SIS structures requires overcoming many materials science and technological challenges to achieve good superconducting properties are SRF performance while providing optimal stoichiometry and morphology of the layers and the interfaces and transparency of grain 
boundaries to extremely high RF current densities. In this work we report results on growth and characterizations of $\mathrm{Nb}_{3} \mathrm{Sn} / \mathrm{Al}_{2} \mathrm{O}_{3}$ multilayers which exhibit good superconducting properties and low-field SRF performance on par with the cavity-grade $\mathrm{Nb}$.

\section{Results and discussion}

Multilayer growth We developed a technique of high-temperature confocal sputtering of $\mathrm{Nb}$ and $\mathrm{Sn}$ from elemental targets to grow stoichiometric $\mathrm{Nb}_{3} \mathrm{Sn}$ multilayers with $\mathrm{Al}_{2} \mathrm{O}_{3}$ interlayers. Details are given in the Supplemental Information. Thin films and multilayers of different thicknesses were grown on different sapphire single crystal substrates for the subsequent characterizations. For instance, $60 \mathrm{~nm}$ thick $\mathrm{Nb}_{3} \mathrm{Sn}$ films were grown on 10 x $10 \mathrm{~mm}$ sapphire substrates for transport, scanning tunneling spectroscopy and electron microscopy characterizations. For RF tests, we grew $\mathrm{Nb}_{3} \mathrm{Sn} / \mathrm{Al}_{2} \mathrm{O}_{3}$ multilayers on $2 "$ diameter sapphire wafers (R-plane, $300 \mu \mathrm{m}$ thick). These multilayers had up to three $60 \mathrm{~nm} \mathrm{Nb} 3 \mathrm{Sn}$ layers separated by $6 \mathrm{~nm} \mathrm{Al}_{2} \mathrm{O}_{3}$. The thickness of the $\mathrm{Nb}_{3} \mathrm{Sn}$ layers was chosen to be smaller than the London penetration depth ${ }^{5,6}$. A $200 \mathrm{~nm}$ thick $\mathrm{Nb}$ film was deposited on the backside of the wafers to prevent leakage of RF field during cavity measurements. The geometry of multilayer samples used in our RF measurements of quality factors is shown in Figure 1.

The $\mathrm{Nb}-\mathrm{Sn}$ phase diagram contains several line compounds. For instance, $\mathrm{Nb}_{3} \mathrm{Sn}$ and $\mathrm{Nb}_{6} \mathrm{Sn}_{5}$ coexist in the region marked in Figure 2a. Here a low- $\mathrm{T}_{c} \mathrm{Nb}_{6} \mathrm{Sn}_{5}$ phase is clearly undesirable in these films ${ }^{21}$. Within the $\mathrm{Nb}_{3} \mathrm{Sn}$ phase region extending from $17-25 \% \mathrm{Sn}$, the critical temperature $\mathrm{T}_{\mathrm{c}}$ degrades steeply as stoichiometry moves away from a 3:1 ratio ${ }^{22}$. These two conditions demand that $\mathrm{Nb}_{3} \mathrm{Sn}$ films should contain $25 \%$ of $\mathrm{Sn}$. This was accomplished by providing processing conditions reflecting the field in the upper right of the phase diagram in Figure 2a, a two-phase region containing only stoichiometric $\mathrm{Nb}_{3} \mathrm{Sn}$ and liquid $\mathrm{Sn}$. Films were grown by confocal sputtering of $\mathrm{Nb}$ and $\mathrm{Sn}$ from elemental targets. By providing a large over-pressure of $\mathrm{Sn}$ at high growth temperatures, it has been found that the ratio of $\mathrm{Nb}: \mathrm{Sn}$ can be pinned at 3:1. The abundance of Sn drives the material into the two-phase region, where excess $\mathrm{Sn}$ re-evaporates from the film, avoiding the formation of Sn precipitates ${ }^{23,24}$. To achieve the high temperatures $\left(>930{ }^{\circ} \mathrm{C}\right)$ required for this growth, sapphire substrates were heated from behind with a $\mathrm{SiC}$ radiative heater. Radiation passed through the substrate and heated the depositing metal directly. Growth temperature was measured by pyrometer. Details of the film growth are given in the Supplemental information.

A series of films was grown with fixed $\mathrm{Nb}$ flux $(0.7 \AA / \mathrm{s})$ and varying Sn flux (0.4-2.5 $\AA / \mathrm{s})$, and low-temperature resistance measurements were carried out to find the window for this selfregulating adsorption-controlled process. Shown in Figure $2 b$ are the dependencies of the critical temperature $T_{c}$ and transition width $\Delta T_{c}$ on the deposition rate of Sn which clearly saturate at $\sim 1$ $\AA / \mathrm{s}$. Given the dependence of $T_{c}$ on $\mathrm{Sn}$ content in $\mathrm{Nb}_{3} \mathrm{Sn}$, this growth rate roughly corresponds to the boundary between two processing regimes. At lower flux, Sn that is necessary to form stoichiometric $\mathrm{Nb}_{3} \mathrm{Sn}$ evaporates before it can be incorporated into the film. At higher flux, the sufficient $\mathrm{Sn}$ is provided to react with all available $\mathrm{Nb}$, and excess $\mathrm{Sn}$ re-evaporates. 
The dielectric $\mathrm{Al}_{2} \mathrm{O}_{3}$ interlayers were grown after allowing $\mathrm{Nb}_{3} \mathrm{Sn}$ to cool down to $<400{ }^{\circ} \mathrm{C}$, using a single stoichiometric target with RF power at a rate of $1.8 \mathrm{~nm} / \mathrm{min}$ without any further heating applied to the substrate. Depositing under these conditions protects the $\mathrm{SiC}$ heater element from oxygen evolved during the sputtering process and prevents undesired reactions with the $\mathrm{Nb}_{3} \mathrm{Sn}$ surface. This $\mathrm{Nb}_{3} \mathrm{Sn} / \mathrm{Al}_{2} \mathrm{O}_{3}$ stack was then heated again to $900+{ }^{\circ} \mathrm{C}$, which allows the $\mathrm{Al}_{2} \mathrm{O}_{3}$ to crystallize, and the process was repeated to grow heterostructures of up to three $\mathrm{Nb}_{3} \mathrm{Sn}$ layers. The chamber setup and growth steps are depicted in

Figure 3 .

Structural characterization A SIS sample with three $\mathrm{Nb}_{3} \mathrm{Sn}$ layers was prepared for analysis by cross-sectional scanning transmission electron microscopy (STEM). A low-magnification image (

Figure 4a) represents the morphology and nanostructure of the stack. Each $\mathrm{Nb}_{3} \mathrm{Sn}$ layer is polycrystalline with irregular interfaces and grain size is $20-100 \mathrm{~nm}$ along the film surface direction. The $\mathrm{Al}_{2} \mathrm{O}_{3}$ layers conform closely to the layer below but are discontinuous along the $\mathrm{Nb}_{3} \mathrm{Sn} / \mathrm{Al}_{2} \mathrm{O}_{3}$ interface. Despite the repeated thermal cycling during stacking, it appears that the lower layers have not degraded in comparison to the top layer.

The chemical stability of these films is further confirmed by compositional mapping with energy dispersive spectroscopy (EDS) (

Figure $4 \mathrm{~b}$ ). Intensity of the $\mathrm{Al} \mathrm{K} \alpha$ and $\mathrm{O} \mathrm{K} \alpha$ peaks are mapped from the region shown on the left. Although the $\mathrm{Al}_{2} \mathrm{O}_{3}$ layers are not continuous, $\mathrm{Al}$ and $\mathrm{O}$ are confined to the $\mathrm{Al}_{2} \mathrm{O}_{3}$ layers, and do not mix with the $\mathrm{Nb}_{3} \mathrm{Sn}$ layers. The exception to this is the presence of $\mathrm{O}$ at the interface of the topmost $\mathrm{Nb}_{3} \mathrm{Sn}$ film with the atmosphere, where ambient conditions are sufficient to cause a reaction. Note that the slight $\mathrm{O}$ signal in the $\mathrm{Nb}_{3} \mathrm{Sn}$ layers in Figure $4 \mathrm{~b}$ are due to the slight oxidation of the TEM specimen surface. As our RF cavity measurements show, these $\mathrm{Al}_{2} \mathrm{O}_{3}$ layers do not contribute significantly to surface resistance at low fields.

A higher-magnification image of the S-I interface is shown in

Figure $4 \mathrm{c}$. The atomic structure of $\mathrm{Nb}_{3} \mathrm{Sn}$ is well-preserved at the interface, suggesting that there is almost no diffusion or intermixing from the $\mathrm{Al}_{2} \mathrm{O}_{3}$. The lower $\mathrm{Nb}_{3} \mathrm{Sn}$ grain orients the [023] direction normal to the film surface, and this direction is also preserved in the upper $\mathrm{Nb}_{3} \mathrm{Sn}$ grain. This can occur when the upper $\mathrm{Nb}_{3} \mathrm{Sn}$ layer deposits with the same epitaxial relationship to the underlying $\mathrm{Al}_{2} \mathrm{O}_{3}$ as the lower layer has with the $\mathrm{Al}_{2} \mathrm{O}_{3}$ substrate. This structure can also form when a $\mathrm{Nb}_{3} \mathrm{Sn}$ grain nucleates on top of a $\mathrm{Nb}_{3} \mathrm{Sn}$ surface exposed by breaks in the discontinuous $\mathrm{Al}_{2} \mathrm{O}_{3}$ layer. X-ray diffractometry indicates that $\mathrm{Nb}_{3} \mathrm{Sn}$ grains in the second layer have more random crystallographic orientation compared to the first layer (see the Supplemental material).

Superconducting properties. Our dc transport measurements have shown that the $\mathrm{Nb}_{3} \mathrm{Sn}$ films capped with $\mathrm{Al}_{2} \mathrm{O}_{3}$ and annealed with no further deposition exhibit good superconducting 
properties. For instance, the superconducting resistive transitions of a bare $\mathrm{Nb}_{3} \mathrm{Sn}$ film and a $\mathrm{Nb}_{3} \mathrm{Sn} / \mathrm{Al}_{2} \mathrm{O}_{3}$ structure annealed at $900{ }^{\circ} \mathrm{C}$ for 10 minutes are shown in

Figure 5a. Here the critical temperature of the annealed sample is about $0.25 \mathrm{~K}$ higher than $T_{c}$ of the unannealed sample, and residual resistivity ratio (RRR), an indicator of crystalline and metallic quality, is improved from 3.5 to 4.26. On the other hand, $\mathrm{Nb}_{3} \mathrm{Sn}$ films annealed without the $\mathrm{Al}_{2} \mathrm{O}_{3}$ cap, even under high Sn flux to prevent evaporative loss, have degraded superconducting properties compared to an un-annealed film.

The superconducting properties essential for the RF performance were characterized by the scanning tunneling spectroscopy (STS) which measures the differential tunneling conductance $\mathrm{dI} / \mathrm{dV}$ proportional to the quasiparticle density of states (DOS), N(E). Shown in

Figure $5 \mathrm{~b}$ is a representative tunneling spectrum measured in the center of a $\mathrm{Nb}_{3} \mathrm{Sn}$ grain at $4 \mathrm{~K}$. The DOS curves, which clearly show the superconducting gap $\Delta$ at the Fermi surface, were fit using the conventional Dynes model ${ }^{25,26}$ :

$$
N(E)=N_{0} \operatorname{Re}\left[\frac{E-i \Gamma}{\sqrt{(E-i \Gamma)^{2}-\Delta^{2}}}\right]
$$

where the phenomenological parameter $\Gamma$ accounts for the broadening of the DOS peaks due to a finite lifetime of quasiparticles, and $\mathrm{N}_{0}$ is the DOS in the normal state. The fit was done with $\Gamma=$ $0.4 \mathrm{meV}$ and $\Delta \approx 3.1 \mathrm{meV}$, consistent with the conventional gap value for a stoichiometric $\mathrm{Nb}_{3} \mathrm{Sn}$ 4 . The ratio $\Gamma / \Delta \approx 13 \%$ in our samples turns out to be about 2-3 times larger than the values observed by tunneling spectroscopy on 1-2 $\mu \mathrm{m}$ thick $\mathrm{Nb}_{3} \mathrm{Sn}$ films for rf applications ${ }^{27}$ and $\mathrm{Nb}$ coupons $^{28}$. The deviations of the STM data from the Dynes model at low energies $E<\Delta$ may indicate the effects of local non-stoichiometry, gap anisotropy and strain ${ }^{22}$, scattering of quasiparticles on magnetic impurities, and a thin layer with deteriorated superconducting properties at the surface $27,28,29,30$. In turn, the subgap quasiparticles states which appear at $|\mathrm{E}|<\Delta$ due to a finite $\Gamma$ contribute to a temperature-independent residual surface resistance $R_{i}$ at $k_{B} T<<$ $\Delta^{5,29}$

$$
R_{i}=\frac{\mu_{0}^{2} \omega^{2} \lambda^{3} \Gamma^{2}}{2 \rho_{n}\left(\Delta^{2}+\Gamma^{2}\right)}
$$

Here $\mu_{0}$ is the permeability of free space, $\rho_{n}$ is the normal-state resistivity, $\lambda$ is the magnetic penetration depth, and $\omega=2 \pi f$ is the circular RF frequency ${ }^{5}$. For $\lambda=120 \mathrm{~nm}, \rho_{n}=3.0 \times 10^{-7} \Omega \mathrm{m}$, and the fit parameters $\Delta=3.1 \mathrm{meV}$ and $\Gamma=0.4 \mathrm{meV}$, we obtain $R_{i} \approx 5.0 \mathrm{n} \Omega$ at f $=1.3 \mathrm{GHz}$. This estimate is of the order of $R_{i} \approx 5-10 \mathrm{n} \Omega$ for large-grain $\mathrm{Nb}$ cavities ${ }^{31}$. Below a few nm thick surface layer but well within the rf penetration depth $\lambda \approx 120 \mathrm{~nm}$, the gap peaks in the DOS are likely much sharper. There are other essential contributions to $R_{i}$ most notably due to non-stoichiometric regions in the bulk ${ }^{27}$, grain boundaries and trapped vortices ${ }^{32}$. 
Low-field RF characterization Multilayer samples grown on 2" sapphire wafers were tested in a hemispherical Nb-coated cavity at SLAC National Accelerator Laboratory. The experimental setup was described previously ${ }^{33}$. A rendering of this cavity is shown in Figure 6bError! Reference source not found. The cavity operates in a $\mathrm{TE}_{032}$-like mode at $11.4 \mathrm{GHz}$, with a pocket on the flat face for mounting 2"-diameter samples (shown in purple). The overall cavity quality factor is measured, and the properties of the wafer can be deduced by comparison with known samples. The geometry of the cavity is engineered such that the magnetic field is strongest at the sample surface, limiting the contribution of the cavity material to the overall cavity loss. According to simulations, the participation factor is 0.33 for the 2"-diameter sample. Crucially, the magnetic field at the sample is in the radial direction and parallel to the sample surface, making it possible to measure RF properties of the sample without interference from the perpendicular component of the field.

The SRF performance of two $\mathrm{Nb}_{3} \mathrm{Sn}$ samples were compared in this system to a cavity-grade bulk $\mathrm{Nb}$ coupon. A $500 \mathrm{~nm}(\sim 4 \lambda) \mathrm{Nb}_{3} \mathrm{Sn}$ film intended to completely screen out the RF magnetic field, and a $3 \times 60 \mathrm{~nm} \mathrm{Nb} 3 \mathrm{Sn}_{2} / \mathrm{Al}_{2} \mathrm{O}_{3}$ trilayer were tested under the RF field. Both samples were coated with a $200 \mathrm{~nm} \mathrm{Nb}$ film on the backside of the wafer to prevent leakage of magnetic field as shown in Figure 1. The quality factor of the cavity with each sample, measured at low power with a network analyzer, is plotted in Figure 6a. The abrupt increase in $\mathrm{Q}$ at about $15 \mathrm{~K}$ corresponds to the superconducting transition of $\mathrm{Nb}_{3} \mathrm{Sn}$, followed by an increase of $\mathrm{Q}(\mathrm{T})$ at $T_{c}=9 \mathrm{~K}$ of the $\mathrm{Nb}$ coated host cavity.

As shown in

Figure $6 \mathrm{a}$, the thick $\mathrm{Nb}_{3} \mathrm{Sn}$ film and the trilayer have nearly identical $\mathrm{Q}$ at $\mathrm{T}<9 \mathrm{~K}$, indicating that $\mathrm{Al}_{2} \mathrm{O}_{3}$ dielectric layers and interfaces do not contribute significantly to the RF dissipation. We would expect the thick $\mathrm{Nb}_{3} \mathrm{Sn}$ film to have a higher $\mathrm{Q}$, as magnetic field is more fully screened before reaching the substrate and backside, so this result suggests that the maximum $\mathrm{Q}$ of these films and multilayers is limited by the quality of the $\mathrm{Nb}_{3} \mathrm{Sn}$ material rather than by the interfaces with $\mathrm{Al}_{2} \mathrm{O}_{3}$. The quality factors of both the film and the trilayer samples exceed $\mathrm{Q}(\mathrm{T})$ of $\mathrm{Nb}$ at $\mathrm{T}>$ $6 \mathrm{~K}$ due to the higher $T_{c}$ of $\mathrm{Nb}_{3} \mathrm{Sn}$ and is about 2 times smaller than $\mathrm{Q}$ of $\mathrm{Nb}$ at $4 \mathrm{~K}$.

Discussion The results of this work show that, despite the obvious non-stoichiometry and inhomogeneity of superconducting properties, grain boundaries, $\mathrm{Nb}$ inclusions, and incomplete $\mathrm{Al}_{2} \mathrm{O}_{3}$ layers, our multilayers exhibit the quality factors on par of those of cavity-grade bulk $\mathrm{Nb}$ at $4 \mathrm{~K}$ and low RF power. The significant local non-stoichiometry of thick (a few micron) polycrystalline $\mathrm{Nb}_{3} \mathrm{Sn}$ coatings of $\mathrm{Nb}$ cavities ${ }^{20,27}$, as well as $\mathrm{Sn}$ depletion at grain boundaries in $\mathrm{Nb}_{3} \mathrm{Sn}^{34-37}$ have been well documented in the literature. Yet, despite these materials issues which are also characteristic of 1-3 $\mu \mathrm{m}$ thick $\mathrm{Nb}_{3} \mathrm{Sn}$ films used in SRF cavities ${ }^{38}$, our $\mathrm{Nb}_{3} \mathrm{Sn}$ SIS structures exhibit higher low-field $\mathrm{Q}$ values than $\mathrm{Nb}$ at $\mathrm{T}>6 \mathrm{~K}{ }^{20}$, consistent with the larger superconducting energy gap $\Delta_{\mathrm{Nb} 3 \mathrm{Sn}} \approx 2 \Delta_{\mathrm{Nb}}$ and a lower BCS surface resistance $R_{B C S} \propto$ $\omega^{2} \rho_{n}^{1 / 2} e^{-\Delta / k_{B} T}$ of $\mathrm{Nb}_{3} \mathrm{Sn}$. These experimental results not only show a remarkable resilience of 
low-field quality factors of $\mathrm{Nb}_{3} \mathrm{Sn}$ to the significant non-stoichiometry and materials imperfections but also suggest that the SRF performance of $\mathrm{Nb}_{3} \mathrm{Sn}$ coatings can be further improved by materials treatments. Our $\mathrm{Nb}_{3} \mathrm{Sn}$ multilayers exhibit a similar resilience of the low-power SRF performance to the materials imperfections.

The slopes of $\mathrm{Q}(\mathrm{T})$ for both the $\mathrm{Nb}_{3} \mathrm{Sn}$ film and multilayer shown in Fig. 5 tend to level off at 4-5 $\mathrm{K}$ and are clearly smaller than the slope of $\mathrm{Q}(\mathrm{T})$ for $\mathrm{Nb}$. This indicates that $\mathrm{Q}(\mathrm{T})$ of the $\mathrm{Nb}_{3} \mathrm{Sn}$ samples at $\mathrm{T}=4-5 \mathrm{~K}$ is not limited by the BCS surface resistance for which the slope of $\mathrm{Q}(\mathrm{T}) \propto$ $e^{\Delta / k_{B} T}$ for $\mathrm{Nb}_{3} \mathrm{Sn}$ would be larger than for $\mathrm{Nb}$ because $\Delta_{\mathrm{Nb} 3 \mathrm{Sn}} \approx 2 \Delta_{\mathrm{Nb}}$. The behavior of $\mathrm{Q}(\mathrm{T})$ of the $\mathrm{Nb}_{3} \mathrm{Sn}$ samples at $4-5 \mathrm{~K}$ is thus indicative of a significant residual surface resistance caused by the multiphase structure of the films and multilayers and trapped vortices. Yet $Q_{0} \simeq 10^{7}$ observed on our $\mathrm{Nb}_{3} \mathrm{Sn}$ multilayers at $11.4 \mathrm{GHz}$ and $4 \mathrm{~K}$ suggests values of $\mathrm{Q}_{0} \sim 10^{9}$ at $4 \mathrm{~K}$ and $1 \mathrm{GHz}$ given the frequency dependence $\mathrm{Q} \propto \omega^{-2}$ which comes from the BCS surface resistance ${ }^{1-3}$, ohmic losses in metallic precipitates smaller than the RF skin depth and perhaps Josephson vortices trapped on grain boundaries ${ }^{39}$.

SRF performance at high RF fields and breakdown fields of $\mathrm{Nb}_{3} \mathrm{Sn} / \mathrm{Al}_{2} \mathrm{O}_{3}$ multilayers are yet to be explored. Generally, the effects of nonstoichiometry, proximity-coupled normal precipitates and weakly-coupled grain boundaries become more pronounced at higher RF fields. For instance, nonstoichiometric grain boundaries in $\mathrm{Nb}_{3} \mathrm{Sn}$ have been identified as prime pinning centers for vortices in $\mathrm{Nb}_{3} \mathrm{Sn}$ wires for high-field dc magnets ${ }^{39}$. However, weakly-coupled grain boundaries in $\mathrm{Nb}_{3} \mathrm{Sn}$ coating layers would block RF currents and cause dissipative penetration of Josephson vortices at fields well below the superheating field ${ }^{40}$, and sub-stoichiometric regions in $\mathrm{Nb}_{3} \mathrm{Sn}$ coated $\mathrm{Nb}$ cavities are suspected to play an important role in $\mathrm{RF}$ cavity quench ${ }^{27}$. At the same time, significant meandering and breaks in $\mathrm{Al}_{2} \mathrm{O}_{3}$ layers shown in Figure 4 may not be detrimental for SRF performance as the layers can still provide their main role of intercepting and pinning small vortex loops originating at surface structural defects ${ }^{5,8}$ since the pinholes sizes $10-50 \mathrm{~nm}$ in the $\mathrm{Al}_{2} \mathrm{O}_{3}$ layers are smaller than magnetic size of the vortex $\lambda \simeq 100-200 \mathrm{~nm}$ of $\mathrm{Nb}_{3} \mathrm{Sn}$. Though $\mathrm{Al}_{2} \mathrm{O}_{3}$ layers do not fully separate $\mathrm{Nb}_{3} \mathrm{Sn}$ layers, we found that a $500 \mathrm{~nm}$ thick $\mathrm{Nb}_{3} \mathrm{Sn}$ film had a quality factor identical to a multilayer with three $60 \mathrm{~nm} \mathrm{Nb}_{3} \mathrm{Sn}$ layers separated by $6 \mathrm{~nm}$ $\mathrm{Al}_{2} \mathrm{O}_{3}$, and both had $\mathrm{Q}$ approximately $2 \mathrm{x}$ lower than a cavity-grade $\mathrm{Nb}$ reference. This is strong evidence that the $\mathrm{Al}_{2} \mathrm{O}_{3}$ and the oxide-metallic interfaces do not contribute to surface resistance samples prepared with this process.

\section{Conclusions}

In summary, we have developed a self-regulating, adsorption-controlled process for growth of $\mathrm{Nb}_{3} \mathrm{Sn}$ films and $\mathrm{Nb}_{3} \mathrm{Sn} / \mathrm{Al}_{2} \mathrm{O}_{3}$ multilayers. We have produced and characterized multiple multilayer samples with up to four superconducting layers. Despite the detrimental effects of nonstoichiometry, grain boundaries and breaks in the meandering $\mathrm{Al}_{2} \mathrm{O}_{3}$ interlayers, the SRF performance of our multilayers turned out to be on par with that of $\mathrm{Nb}$ films. The growth technique 
reported in this work provides a platform for further optimizations of the SRF properties of SIS high-performance multilayers for superconducting resonator applications.

\section{Methods}

$\mathrm{Nb}_{3} \mathrm{Sn}$ films were sputtered from elemental $\mathrm{Nb}(99.95 \%)$ and $\mathrm{Sn}(99.99 \%)$ targets in 3 mTorr of Ar at a distance of $15.5 \mathrm{~cm}$ from the substrate. DC power to the sputter guns was current-controlled, and deposition rate was measured with an in situ quartz crystal monitor prior to growth. Pyrometer reading of the $\mathrm{SiC}$ heating element at the beginning of growth was $\sim 1250{ }^{\circ} \mathrm{C}$, and dropped to around $905^{\circ} \mathrm{C}$ after $60 \mathrm{~nm}$ was deposited. $\mathrm{Al}_{2} \mathrm{O}_{3}$ was sputtered from a stoichiometric 2 " diameter ceramic target after the pyrometer reading fell below $400{ }^{\circ} \mathrm{C}$. After deposition, the temperature was ramped back up to a pyrometer reading of $905^{\circ} \mathrm{C}$ over the course of 10 minutes. These two steps were repeated to produce the multilayers.

Scanning transmission electron microscope (STEM) imaging and elemental analysis were performed in a probe-corrected JEOL JEM-ARM200cF with an Oxford X-Max ${ }^{\mathrm{N}}$ 100TLE SDD energy dispersive X-ray spectroscopy (EDS) detector.

Superconducting transitions were measured in a closed-loop He cooler using 4-point van der Pauw geometry on 10x10 mm samples. The critical temperature $T_{c}$ is defined as the temperature at which the sheet resistance falls below $1 \%$ of its normal state value at $18 \mathrm{~K}$. The transition width $\Delta T_{c}$ is defined as a difference between $T_{c}$ and the point at which the lines drawn through the normal-state resistance and transition region intersect.

Low-temperature scanning tunneling microscopy/spectroscopy (STM/S) measurements were carried out in a Unisoku-1300 STM system at 4K using polycrystalline PtIr tips. The dI/dV spectra were acquired using standard lock-in technique by applying a bias modulation of $0.2 \mathrm{mV}$ (r.m.s.) at $732 \mathrm{~Hz}$.

\section{Acknowledgement}

This work was supported by the US Department of Energy under grant \# DE-SC0010081-020. Work at West Virginia University was supported by the U.S. Department of Energy under Award \# DE-SC0017632. Work at SLAC National Accelerator Laboratory was supported by the U.S. Department of Energy under contract \# DE-AC02-76SF00515. The nanostructural characterizations were supported by the National High Magnetic Field Laboratory and the National Science Foundation under grant \# NSF/DMR-1644779), and by the State of Florida.

\section{Author contributions}

C.S. developed multilayer growth process and fabricated samples. J.M. participated in RF characterization. Y.S., F.K., and L.X. performed cross-sectional TEM characterization. H.Z. and L.L. performed low-temperature STM characterization. P.B.W. performed low-temperature RF 
surface resistance characterization. C.B.E and A.G. initiated and directed the project. C.S and A.G. wrote the manuscript with contributions from all authors.

\section{Competing interests}

The authors declare no competing interests.

\section{Data availability}

The data that supports the findings of the work are in the manuscripts main text and Supplementary Information. Additional data are available from the corresponding author upon reasonable request. 


\section{References}

1. Padamsee, H., Knobloch, J. \& Hays, T. RF Superconductivity for Accelerators. (Wiley New York, 2008).

2. Gurevich, A. Superconducting Radio-Frequency Fundamentals for Particle Accelerators. Rev. Accel. Sci. Technol. 05, 119-146 (2012).

3. Padamsee, H. S. Superconducting Radio-Frequency Cavities. Annu. Rev. Nucl. Part. Sci. 64, 175-196 (2014).

4. Valente-Feliciano, A.-M. Superconducting RF materials other than bulk niobium: a review. Supercond. Sci. Technol. 29, 113002 (2016).

5. Gurevich, A. Theory of RF superconductivity for resonant cavities. Supercond. Sci. Technol. 30, 034004 (2017).

6. Gurevich, a. Enhancement of rf breakdown field of superconductors by multilayer coating. Appl. Phys. Lett. 88, 012511 (2006).

7. Kubo, T., Iwashita, Y. \& Saeki, T. Radio-frequency electromagnetic field and vortex penetration in multilayered superconductors. Appl. Phys. Lett. 104, 032603 (2014).

8. Gurevich, A. Maximum screening fields of superconducting multilayer structures. AIP $A d v .5,017112$ (2015).

9. Liarte, D. B. et al. Theoretical estimates of maximum fields in superconducting resonant radio frequency cavities: stability theory, disorder, and laminates. Supercond. Sci. Technol. 30, 033002 (2017).

10. Kubo, T. Multilayer coating for higher accelerating fields in superconducting radiofrequency cavities: a review of theoretical aspects. Supercond. Sci. Technol. 30, 023001 (2017).

11. Antoine, C. Z. et al. Characterization of superconducting nanometric multilayer samples for superconducting rf applications: First evidence of magnetic screening effect. Phys. Rev. Spec. Top. - Accel. Beams 13, 121001 (2010).

12. Tajima, T. et al. Studies on thin film $\mathrm{MgB}_{2}$ for applications to RF structures for particle accelerators. in AIP Conference Proceedings 1435, 297-304 (2012).

13. Antoine, C. Z., Villegier, J.-C. \& Martinet, G. Study of nanometric superconducting multilayers for RF field screening applications. Appl. Phys. Lett. 102, 102603 (2013).

14. Beringer, D. B. et al. Thickness Dependence and Enhancement of $\mathrm{H}_{\mathrm{cl}}$ in Epitaxial $\mathrm{MgB}_{2}$ Thin Films. IEEE Trans. Appl. Supercond. 23, 7500604-7500604 (2013).

15. Roach, W. M., Beringer, D. B., Li, Z., Clavero, C. \& Lukaszew, R. A. Magnetic Shielding Larger Than the Lower Critical Field of Niobium in Multilayers. IEEE Trans. Appl. Supercond. 23, 8600203-8600203 (2013).

16. Tan, T. et al. Enhancement of lower critical field by reducing the thickness of epitaxial and polycrystalline $\mathrm{MgB}_{2}$ thin films. APL Mater. 3, 041101 (2015). 
17. Tan, T., Wolak, M. A., Xi, X. X., Tajima, T. \& Civale, L. Magnesium diboride coated bulk niobium: a new approach to higher acceleration gradient. Sci. Rep. 6, 35879 (2016).

18. Junginger, T., Wasserman, W. \& Laxdal, R. E. Superheating in coated niobium. Supercond. Sci. Technol. 30, 125012 (2017).

19. Antoine, C. Z. et al. Optimization of tailored multilayer superconductors for RF application and protection against premature vortex penetration. Supercond. Sci. Technol. 32, 085005 (2019).

20. Posen, S. \& Hall, D. L. $\mathrm{Nb}_{3} \mathrm{Sn}$ superconducting radiofrequency cavities: fabrication, results, properties, and prospects. Supercond. Sci. Technol. 30, 033004 (2017).

21. Charlesworth, J. P., Macphail, I. \& Madsen, P. E. Experimental work on the niobium-tin constitution diagram and related studies. J. Mater. Sci. 5, 580-603 (1970).

22. Godeke, A. A review of the properties of $\mathrm{Nb}_{3} \mathrm{Sn}$ and their variation with $\mathrm{A} 15$ composition, morphology and strain state. Supercond. Sci. Technol. 19, R68-R80 (2006).

23. Allen, L. H., Anklam, W., Beasley, M. R., Hammond, R. H. \& Turneaure, J. P. RF surface resistance in $\mathrm{Nb}_{3} \mathrm{Sn}$ thin films. IEEE Trans. Magn. 21, 525-527 (1985).

24. Allen, L.H., Beasley, M.R., Hammond, R.H., Turneaure, J.P. RF Surface Resistance of $\mathrm{Nb}_{3} \mathrm{Sn}, \mathrm{NbZr}$, and NbN Thin Films. IEEE Trans. Magn. 23, 1405-1408 (1987).

25. Dynes, R. C., Narayanamurti, V. \& Garno, J. P. Direct Measurement of QuasiparticleLifetime Broadening in a Strong-Coupled Superconductor. Phys. Rev. Lett. 41, 1509-1512 (1978).

26. Dynes, R. C., Garno, J. P., Hertel, G. B. \& Orlando, T. P. Tunneling Study of Superconductivity near the Metal-Insulator Transition. Phys. Rev. Lett. 53, 2437-2440 (1984).

27. Becker, C. et al. Analysis of $\mathrm{Nb}_{3} \mathrm{Sn}$ surface layers for superconducting radio frequency cavity applications. Appl. Phys. Lett. 106, 082602 (2015).

28. Lechner, E. M. et al. Electron Tunneling and X-Ray Photoelectron Spectroscopy Studies of the Superconducting Properties of Nitrogen-Doped Niobium Resonator Cavities. Phys. Rev. Appl. 13, 044044 (2020).

29. Gurevich, A. \& Kubo, T. Surface impedance and optimum surface resistance of a superconductor with an imperfect surface. Phys. Rev. B 96, 184515 (2017).

30. Proslier, T. et al. Tunneling study of cavity grade Nb: Possible magnetic scattering at the surface. Appl. Phys. Lett. 92, 212505 (2008).

31. Visentin, B., Barthe, M. F., Moineau, V. \& Desgardin, P. Involvement of hydrogenvacancy complexes in the baking effect of niobium cavities. Phys. Rev. Spec. Top. - Accel. Beams 13, 052002 (2010).

32. Dhakal, P., Ciovati, G. \& Gurevich, A. Flux expulsion in niobium superconducting radiofrequency cavities of different purity and essential contributions to the flux sensitivity. Phys. Rev. Accel. Beams 23, 023102 (2020). 
33. Welander, P., Franzi, M. \& Tantawi, S. Cryogenic RF characterization of superconducting materials at SLAC with hemispherical cavities. in Proceedings of SRF2015 735-738 (2015).

34. Suenaga, M. \& Jansen, W. Chemical compositions at and near the grain boundaries in bronze-processed superconducting Nb3Sn. Appl. Phys. Lett. 43, 791-793 (1983).

35. Sandim, M. J. R. et al. Grain boundary segregation in a bronze-route $\mathrm{Nb}_{3} \mathrm{Sn}$ superconducting wire studied by atom probe tomography. Supercond. Sci. Technol. 26, 055008 (2013).

36. Lee, J. et al. Atomic-scale analyses of $\mathrm{Nb}_{3} \mathrm{Sn}$ on $\mathrm{Nb}$ prepared by vapor diffusion for superconducting radiofrequency cavity applications: a correlative study. Supercond. Sci. Technol. 32, 024001 (2019).

37. Lee, J. et al. Grain-boundary structure and segregation in $\mathrm{Nb}_{3} \mathrm{Sn}$ coatings on $\mathrm{Nb}$ for highperformance superconducting radiofrequency cavity applications. Acta Mater. 188, 155165 (2020).

38. Spina, T., Tennis, B. M., Lee, J., Seidman, D. N. \& Posen, S. Development and Understanding of $\mathrm{Nb}_{3} \mathrm{Sn}$ films for radiofrequency applications through a sample-host 9cell cavity. Supercond. Sci. Technol. 34, 015008 (2021).

39. Scanlan, R. M., Fietz, W. A. \& Koch, E. F. Flux pinning centers in superconducting $\mathrm{Nb}_{3}$ Sn. J. Appl. Phys. 46, 2244-2249 (1975).

40. Sheikhzada, A. \& Gurevich, A. Dynamic transition of vortices into phase slips and generation of vortex-antivortex pairs in thin film Josephson junctions under dc and ac currents. Phys. Rev. B 95, 214507 (2017). 
Figures Captions

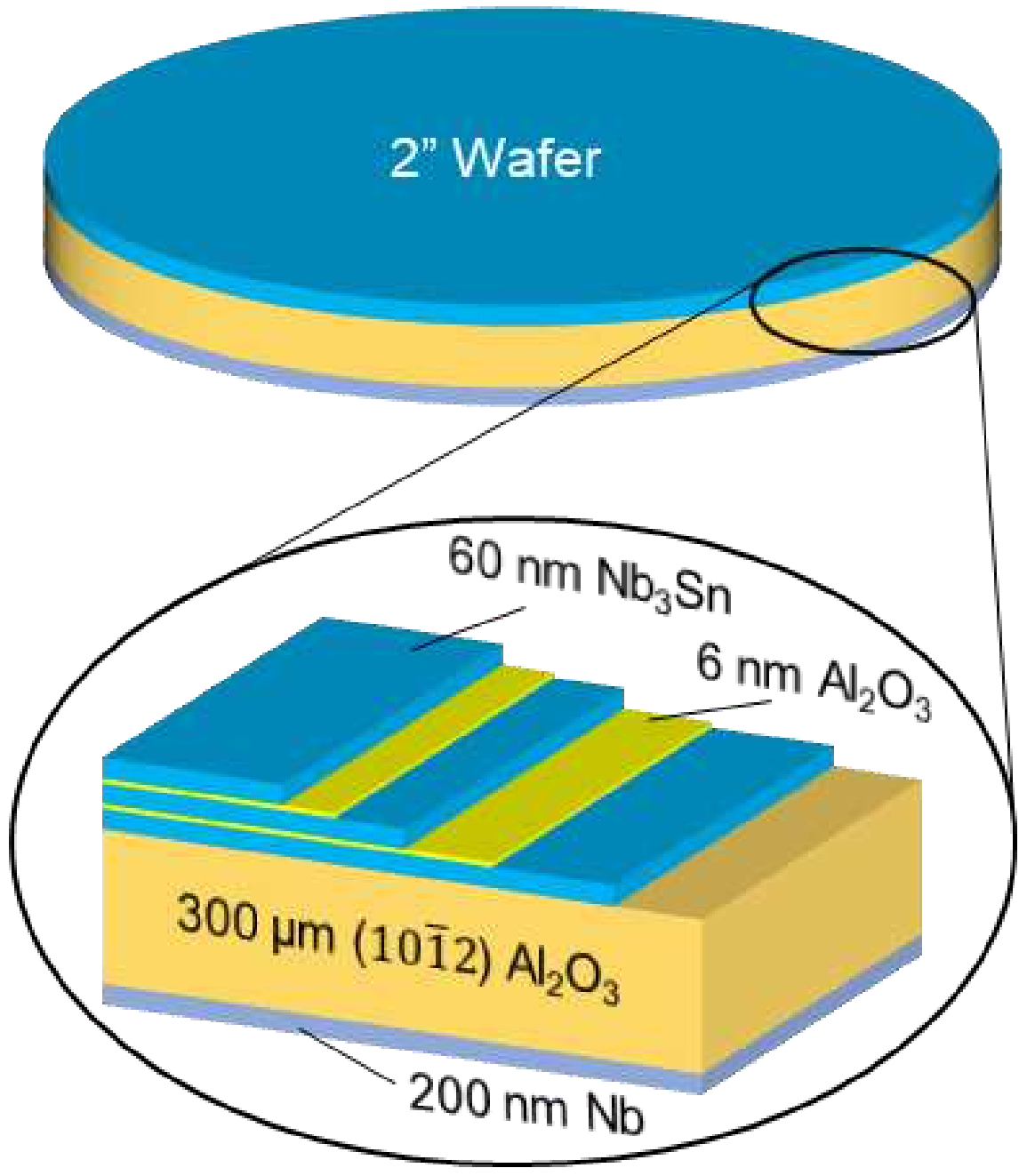

Figure 1. Schematic of $\mathrm{Nb}_{3} \mathrm{Sn} / \mathrm{Al}_{2} \mathrm{O}_{3}$ multilayer heterostructures on $\mathrm{Al}_{2} \mathrm{O}_{3}$ wafer. Back side of $\mathrm{Al}_{2} \mathrm{O}_{3}$ wafer is coated with a thick $\mathrm{Nb}$ film. 
(a)

(b)
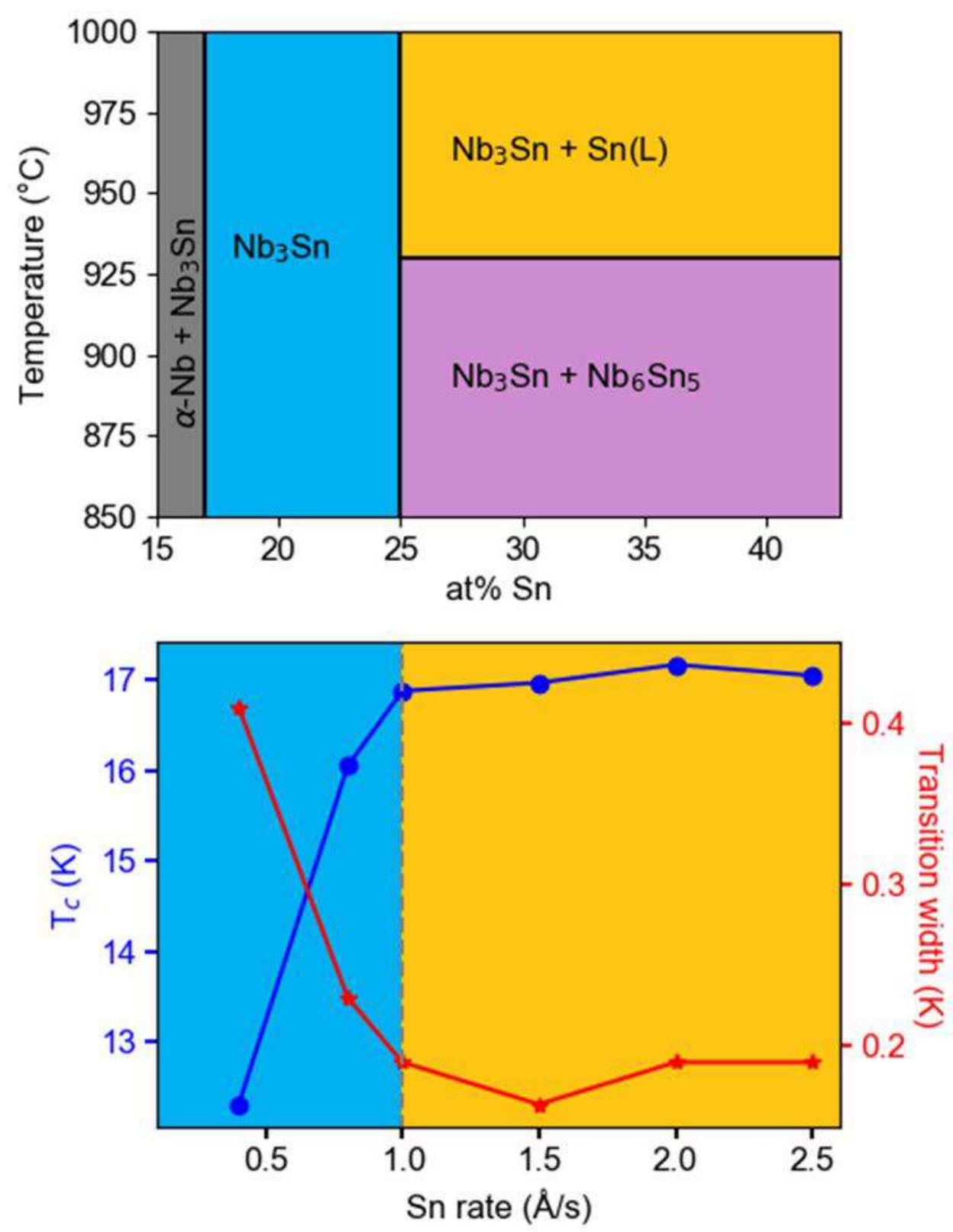

Figure 2. Connecting the $\mathrm{Nb}-\mathrm{Sn}$ phase diagram to electrical properties and process window. (a) Relevant adsorption-controlled growth regime (orange) of $\mathrm{Nb}-\mathrm{Sn}$ phase diagram. $\mathrm{Nb}_{3} \mathrm{Sn}$ spans $17-25 \% \mathrm{Sn}$, and the desired $\mathrm{Nb}_{3} \mathrm{Sn}+$ liquid $\mathrm{Sn}$ field lies above $930{ }^{\circ} \mathrm{C}$ and $25 \% \mathrm{Sn}$. (b) $\mathrm{T}_{\mathrm{c}}, \Delta \mathrm{T}_{\mathrm{c}}$, vs the $\mathrm{Sn}$ flux of $60 \mathrm{~nm}$ thick $\mathrm{Nb}_{3} \mathrm{Sn}$ single layer thin films on $\mathrm{Al}_{2} \mathrm{O}_{3}$ substrates. $\mathrm{T}_{\mathrm{c}}$ levels off above $1.0 \AA / \mathrm{s} \mathrm{Sn}$, corresponding to the adsorption-controlled growth window and the film composition reach $25 \% \mathrm{Sn}$. 


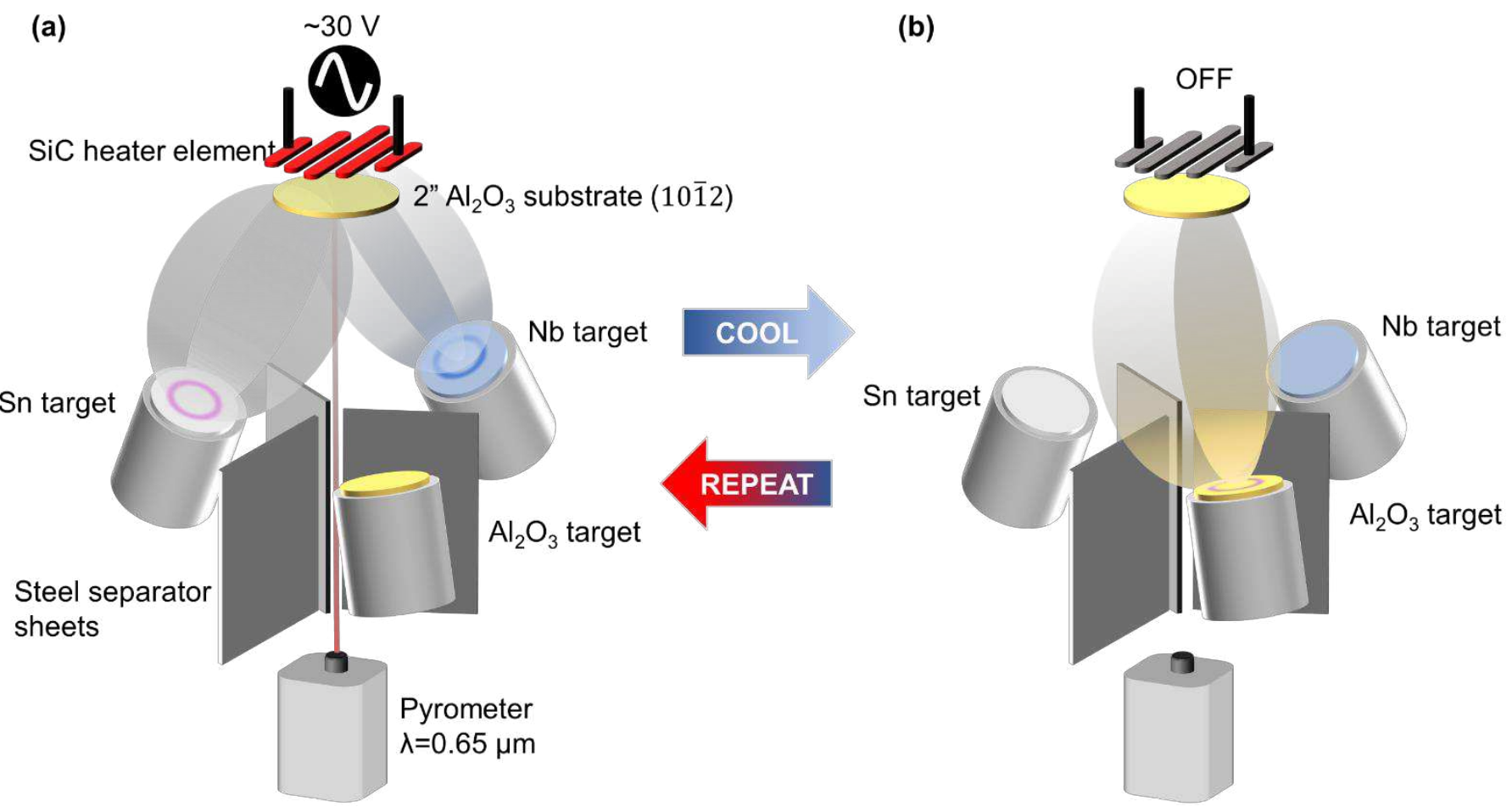

Figure 3. Schematic of thin films deposition setup and sequential processing steps for $\mathrm{Nb}_{3} \mathrm{Sn} / \mathrm{Al}_{2} \mathrm{O}_{3}$ multilayer heterostructures. (a) $\mathrm{Nb}$ and $\mathrm{Sn}$ are sputtered onto $\mathrm{Al}_{2} \mathrm{O}_{3}$ substrate while heater element is powered on. (b) After allowing film to cool, $\mathrm{Al}_{2} \mathrm{O}_{3}$ is sputtered from a single stoichiometric $\mathrm{Al}_{2} \mathrm{O}_{3}$ target. Sample is heated again to anneal $\mathrm{Al}_{2} \mathrm{O}_{3}$. These two steps are repeated to produce multilayer samples. 
(a)

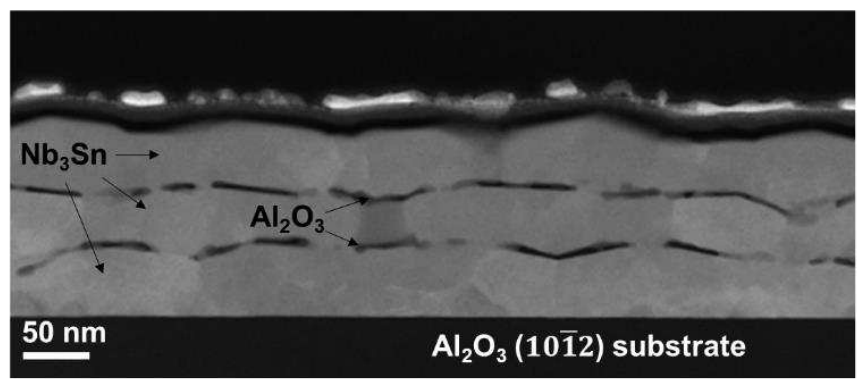

(b)

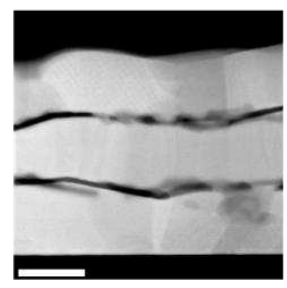

(c)

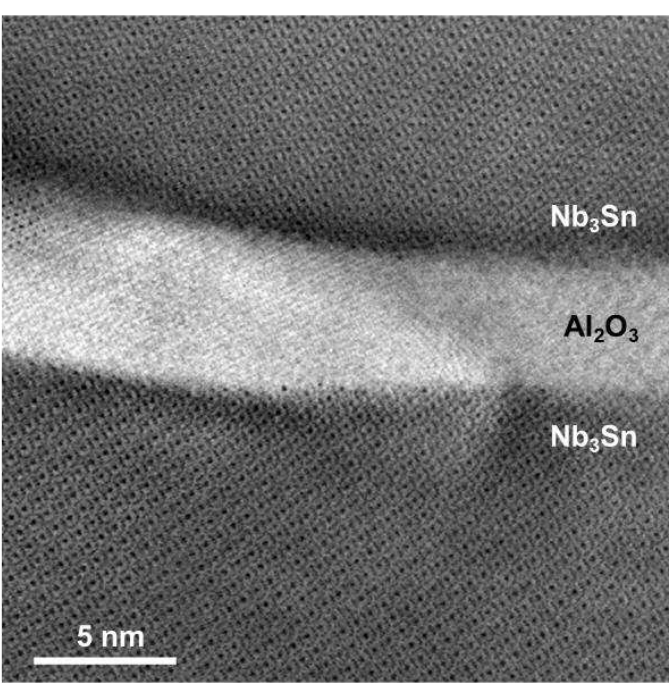

Figure 4. Cross-sectional transmission electron microscopy images of $\mathrm{Nb}_{3} \mathrm{Sn} / \mathrm{Al}_{2} \mathrm{O}_{3}$ multilayer heterostructures (a) Low-magnification image of trilayer morphology. (b) EDS compositional mapping of $\mathrm{Al}$ and $\mathrm{O}$ showing no interdiffusion between $\mathrm{Al}_{2} \mathrm{O}_{3}$ and $\mathrm{Nb}_{3} \mathrm{Sn}$. (c) Highmagnification image of the interfaces between $\mathrm{Al}_{2} \mathrm{O}_{3}$ and $\mathrm{Nb}_{3} \mathrm{Sn}$. 
(a)

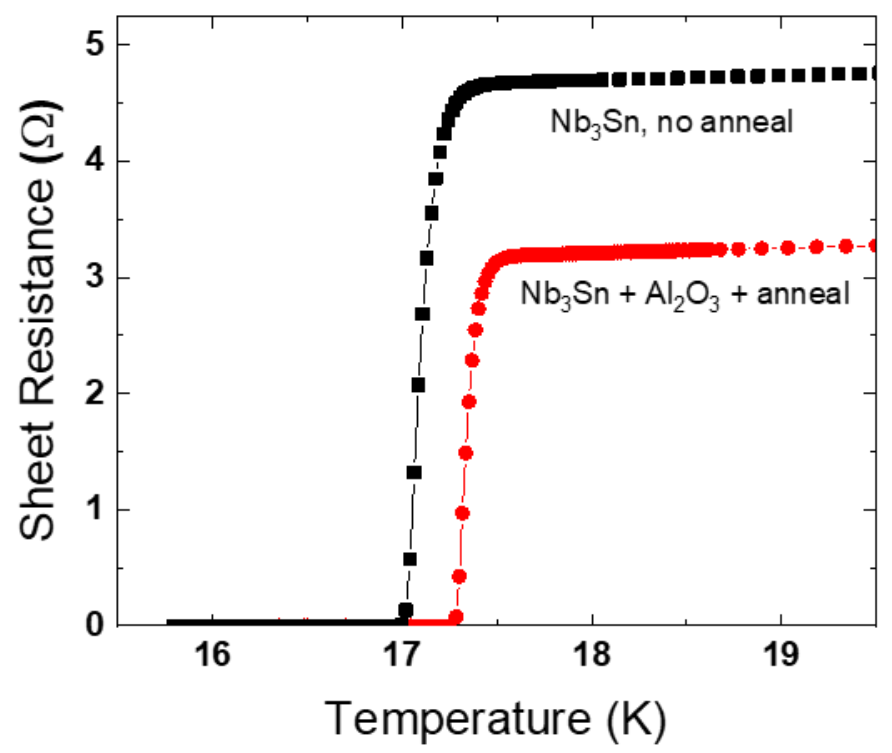

(b)

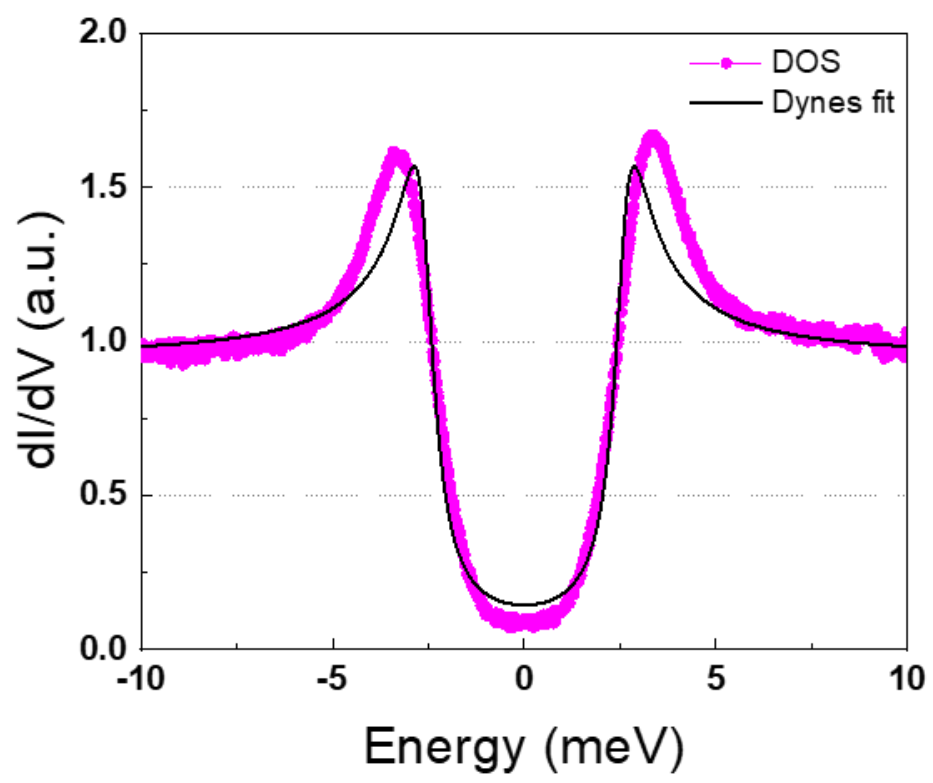

Figure 5. Electrical properties of single-layer $\mathrm{Nb}_{3} \mathrm{Sn}$ films. (a) Resistive superconducting transition for two identical $60 \mathrm{~nm}$ thick films, one of which is capped with $6 \mathrm{~nm} \mathrm{Al} \mathrm{O}_{3}$ overlayer and annealed at $900{ }^{\circ} \mathrm{C}$ for 10 minutes. (b) Density of states measured by scanning tunneling microscopy and Dynes fit for a $60 \mathrm{~nm}$ thick $\mathrm{Nb}_{3} \mathrm{Sn}$ film. 
(a)

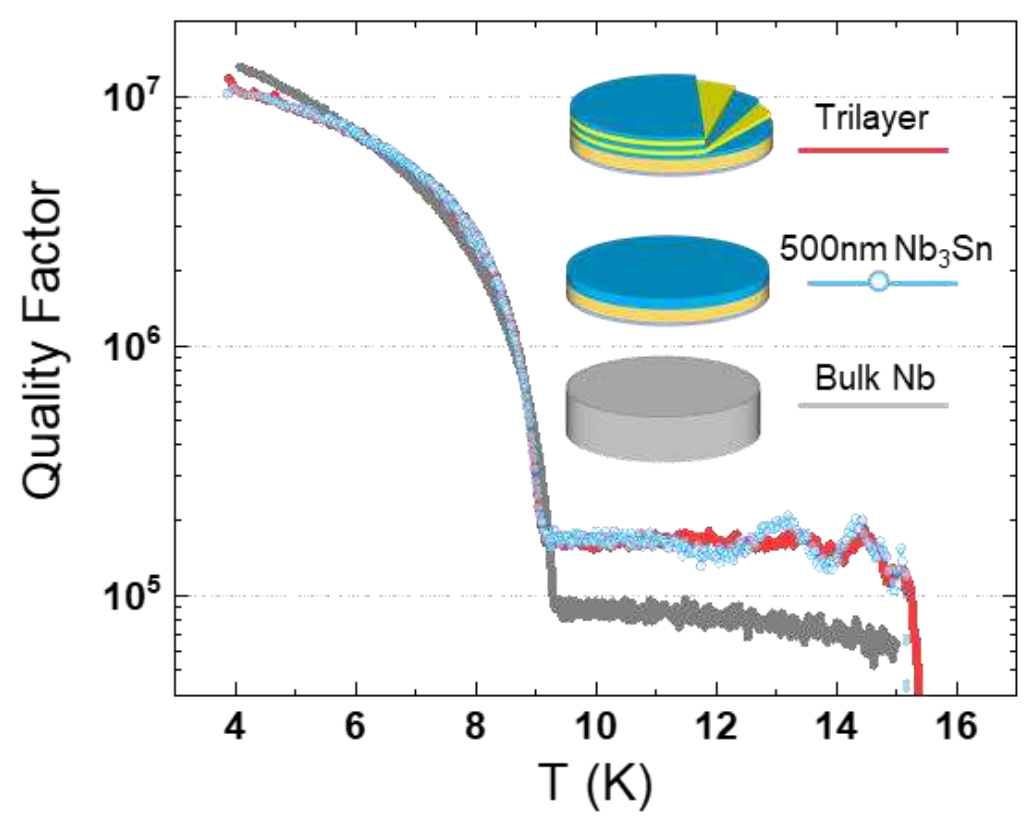

(b)

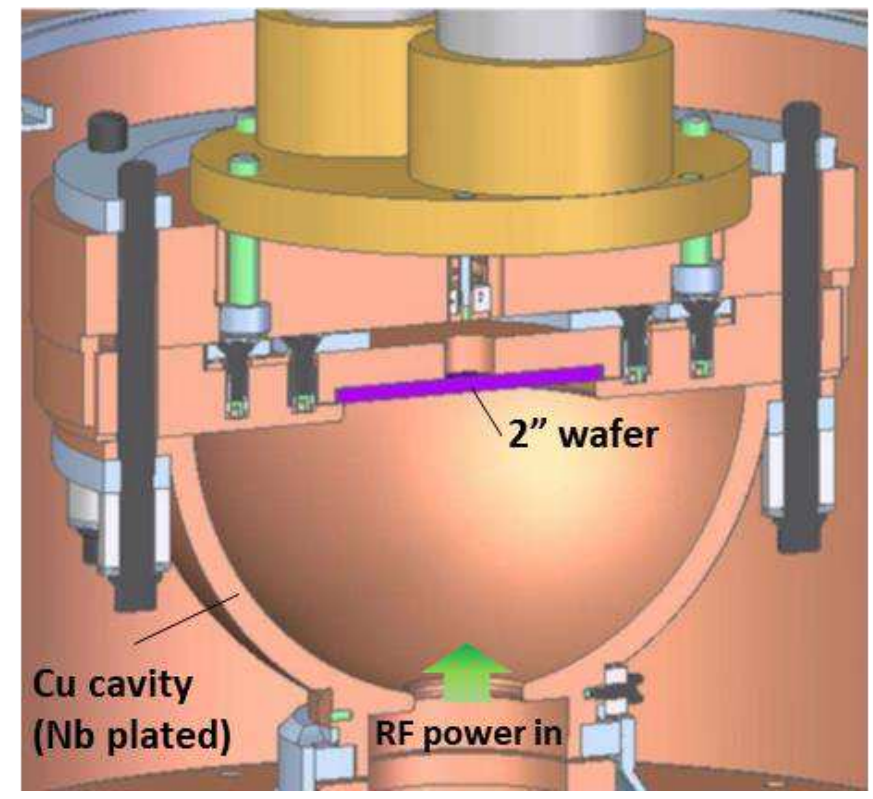

Figure 6. (a) RF Surface resistance measurements of $\mathrm{Nb}_{3} \mathrm{Sn}$ film and multilayer compared to cavity-grade $\mathrm{Nb}$. (b) Cutaway of hemispherical resonator cavity at SLAC used for these measurements. 
Figures

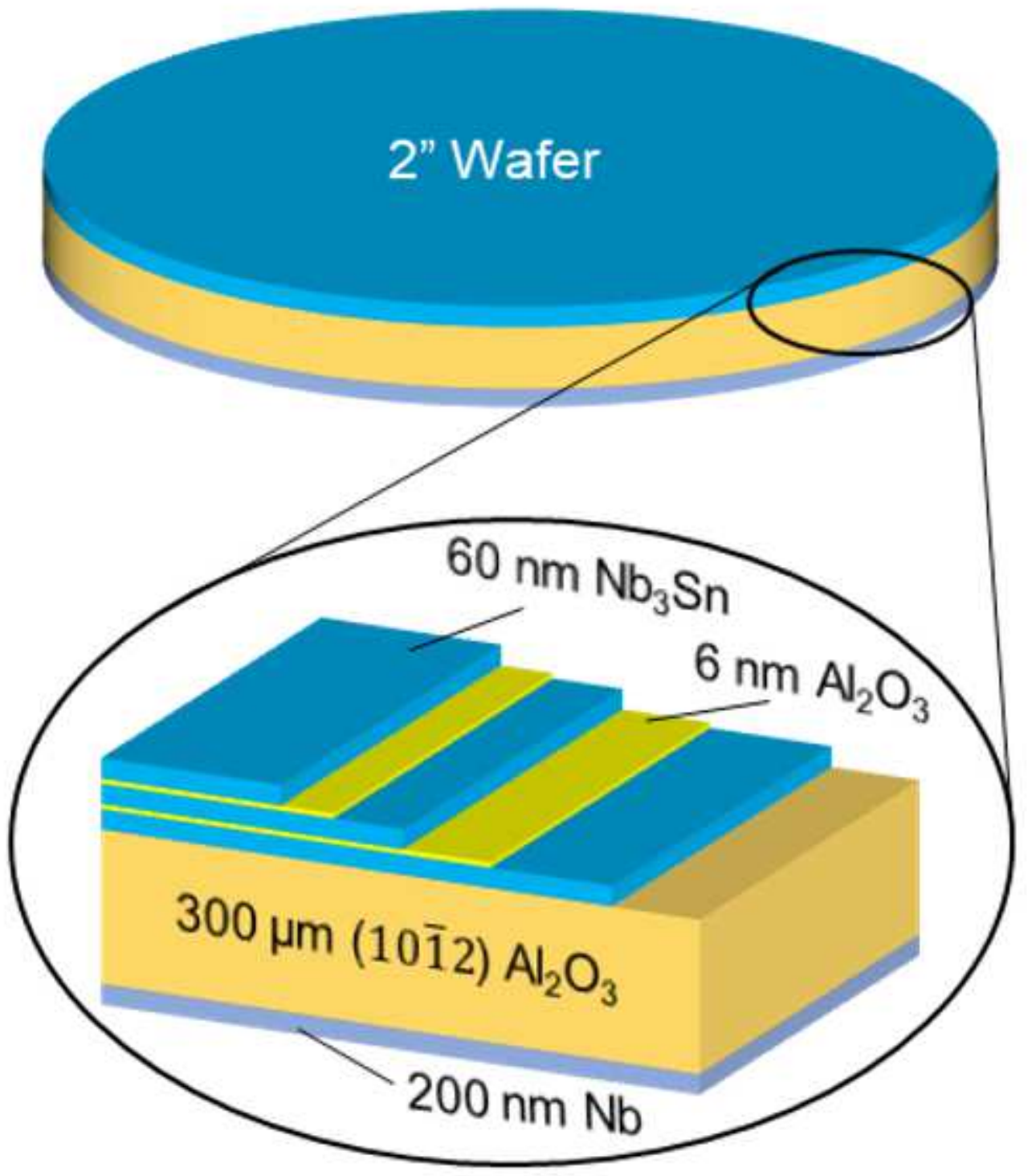

Figure 1

Schematic of Nb3Sn/Al2O3 multilayer heterostructures on Al2O3 wafer. Back side of Al2O3 wafer is coated with a thick $\mathrm{Nb}$ film. 
(a)

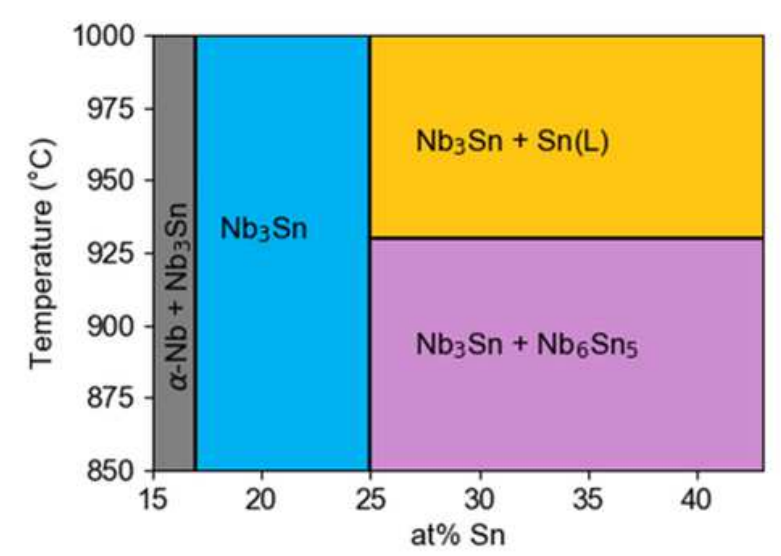

(b)

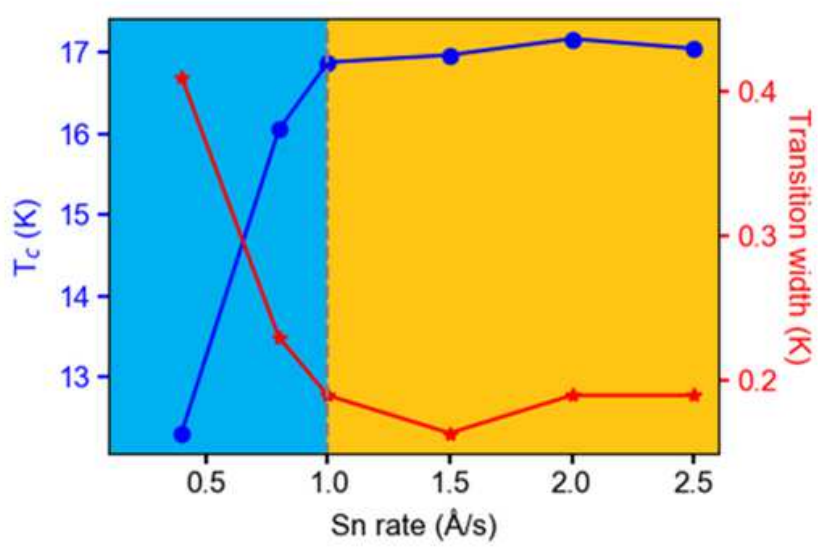

Figure 2

Connecting the $\mathrm{Nb}-\mathrm{Sn}$ phase diagram to electrical properties and process window. (a) Relevant adsorption-controlled growth regime (orange) of $\mathrm{Nb}-\mathrm{Sn}$ phase diagram. Nb3Sn spans $17-25 \% \mathrm{Sn}$, and the desired Nb3Sn + liquid Sn field lies above $930^{\circ} \mathrm{C}$ and $25 \% \mathrm{Sn}$. (b) Tc, $\Delta \mathrm{Tc}$, vs the $\mathrm{Sn}$ flux of $60 \mathrm{~nm}$ thick $\mathrm{Nb} 3 \mathrm{Sn}$ single layer thin films on Al203 substrates. Tc levels off above $1.0 \AA / \mathrm{s} S \mathrm{~S}$, corresponding to the adsorption-controlled growth window and the film composition reach $25 \% \mathrm{Sn}$.

(a)

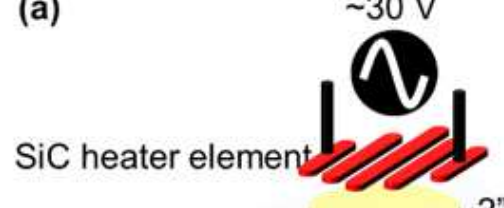

$2 " \mathrm{Al}_{2} \mathrm{O}_{3}$ substrate $(10 \overline{1} 2)$

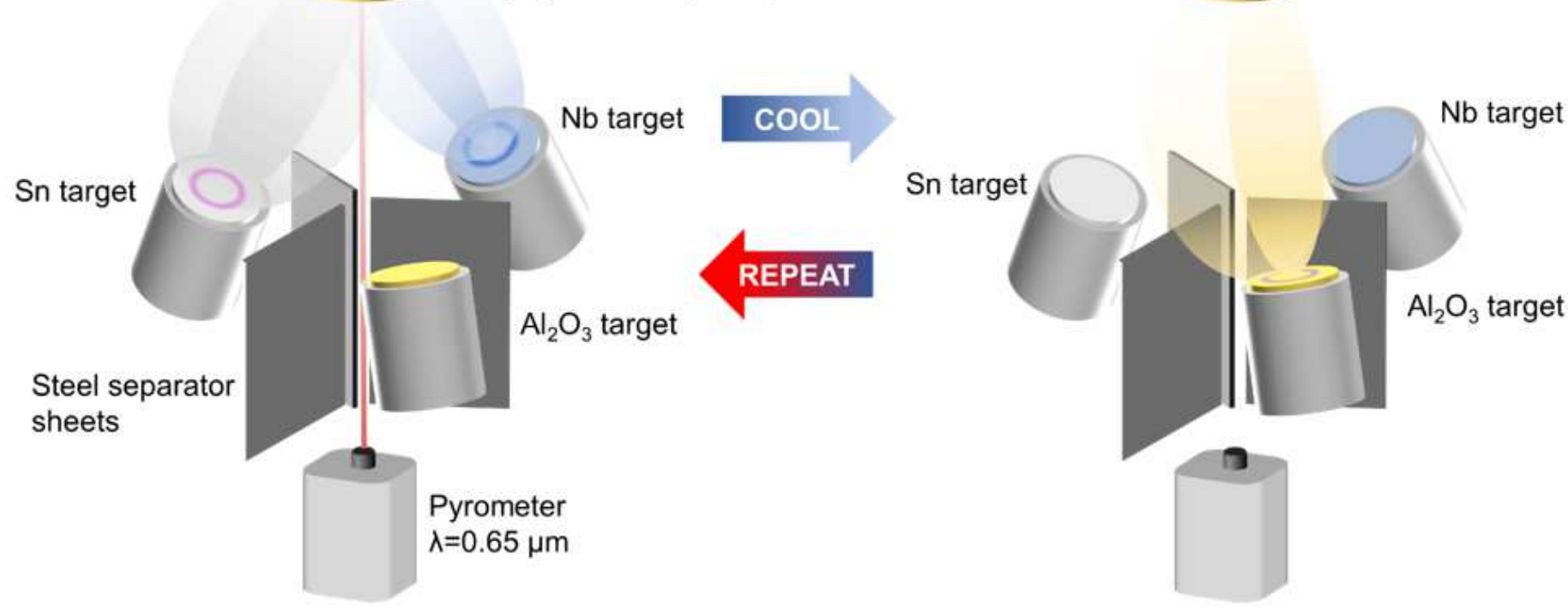

(b)

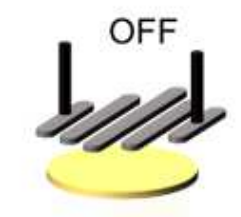

\section{Figure 3}

Schematic of thin films deposition setup and sequential processing steps for Nb3Sn/Al2O3 multilayer heterostructures. (a) $\mathrm{Nb}$ and $\mathrm{Sn}$ are sputtered onto Al2O3 substrate while heater element is powered on. 
(b) After allowing film to cool, Al2O3 is sputtered from a single stoichiometric Al2O3 target. Sample is heated again to anneal Al203. These two steps are repeated to produce multilayer samples.

(a)

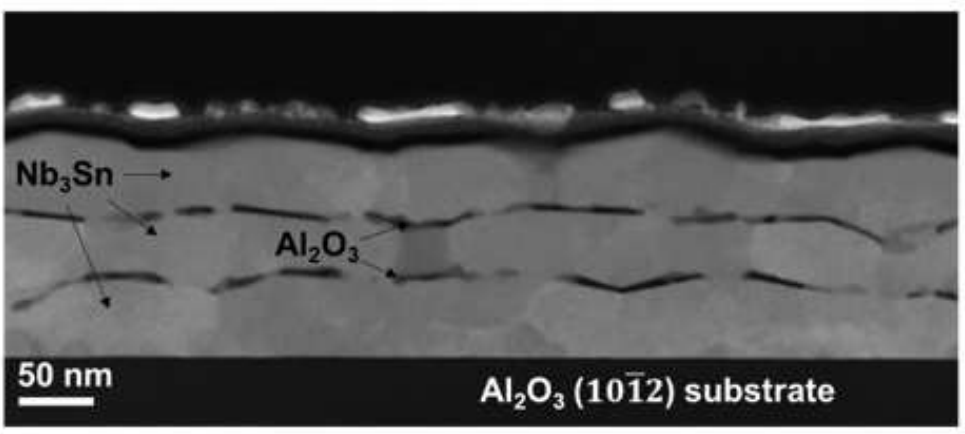

(b)

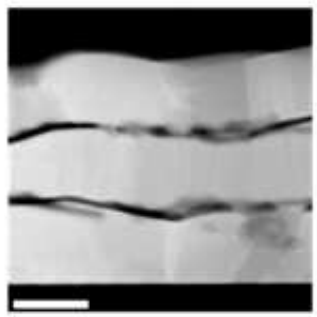

(c)

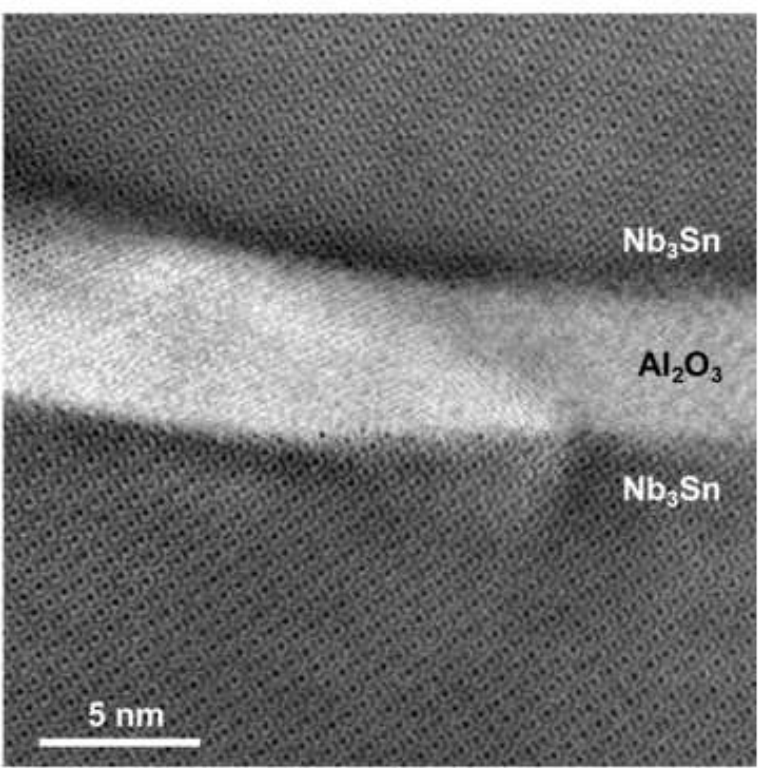

Figure 4

Cross-sectional transmission electron microscopy images of Nb3Sn/Al2O3 multilayer heterostructures (a) Low-magnification image of trilayer morphology. (b) EDS compositional mapping of Al and $\mathrm{O}$ showing no interdiffusion between Al2O3 and Nb3Sn. (c) High-magnification image of the interfaces between Al2O3 and Nb3Sn.

(a)

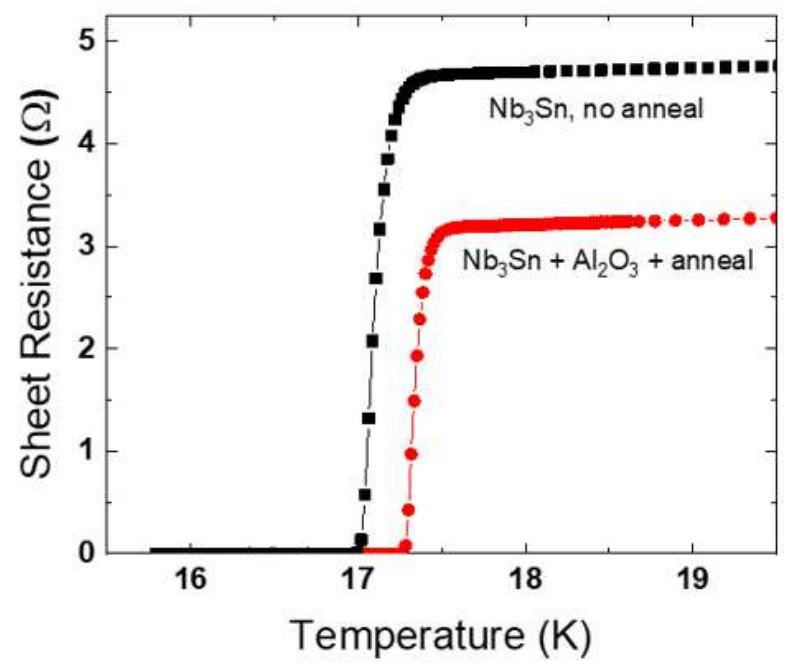

(b)

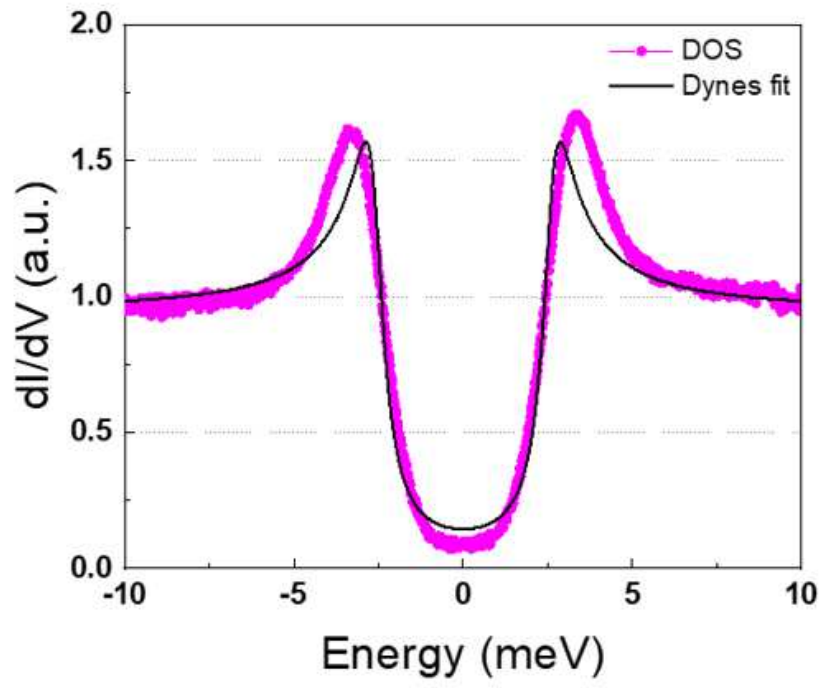

Figure 5 
Electrical properties of single-layer Nb3Sn films. (a) Resistive superconducting transition for two identical $60 \mathrm{~nm}$ thick films, one of which is capped with $6 \mathrm{~nm}$ Al2O3 overlayer and annealed at $900{ }^{\circ} \mathrm{C}$ for 10 minutes. (b) Density of states measured by scanning tunneling microscopy and Dynes fit for a $60 \mathrm{~nm}$ thick Nb3Sn film.

(a)

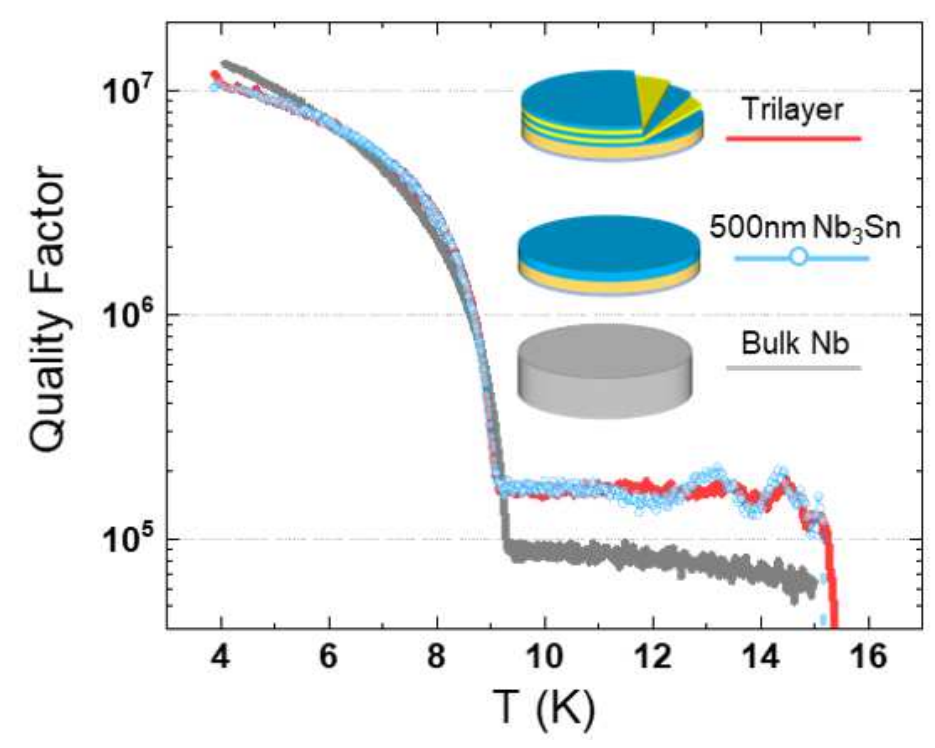

(b)

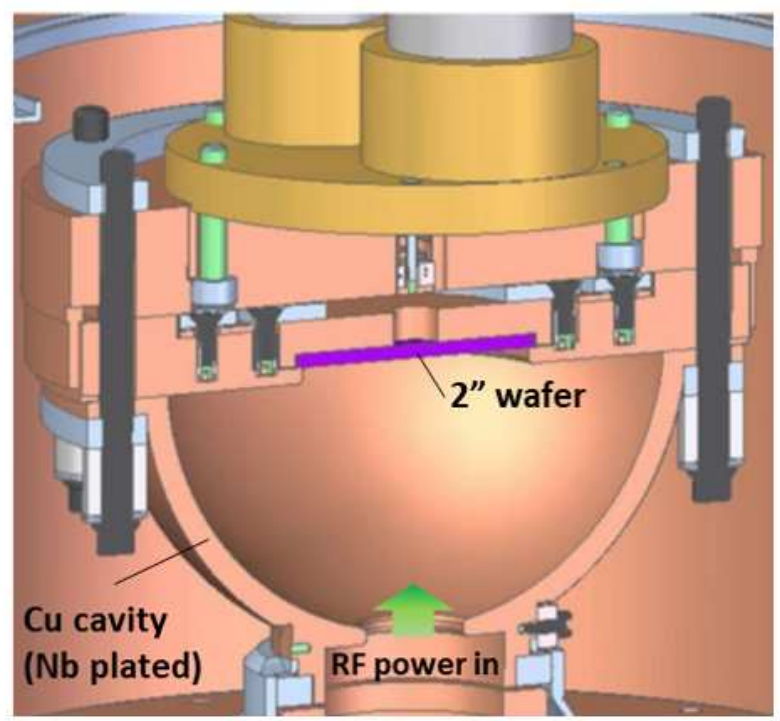

\section{Figure 6}

(a) RF Surface resistance measurements of Nb3Sn film and multilayer compared to cavity-grade Nb. (b) Cutaway of hemispherical resonator cavity at SLAC used for these measurements.

\section{Supplementary Files}

This is a list of supplementary files associated with this preprint. Click to download.

- Nb3SnmultilayerSupplementallnfoFinal.docx 